\title{
ANTIMUTAGENIC EFFECT OF PHENOLIC ACIDS
}

\author{
Lucia Birošováa , Mária Mikulášováa ${ }^{\text {Štefánia Vaverkováb }}$
}

\author{
a Department of Biochemistry and Microbiology, Faculty of Food and Chemical Technology, Slovak University of Technology, \\ Bratislava, Slovakia \\ ${ }^{b}$ Department of Pharmacognosy and Botany, Faculty of Pharmacy, Comenius University, Bratislava, Slovakia \\ e-mail: lucia.birosova@stuba.sk
}

Received: June 10, 2005; Accepted: September 25, 2005

Key words: Phenolic acid/Antimutagenity/Salmonella typhimurium

In the present study, the Salmonella typhimurium tester strain TA 100 was used in the plate-incorporation test to examine the antimutagenic potential of caffeic, ferulic and cichoric acids extracted from plant species of genera Echinacea (L) Moench, as well as of another phenolic acids, on 3-(5-nitro-2-furyl)acrylic acid (5NFAA) and sodium azide mutagenicity. All tested compounds possess antimutagenic activity. In the case of 5NFAA, the antimutagenic potency of tested compounds was in the order of gallic acid > ferulic acid > caffeic acid > syringic acid $>$ vanillic acid. The mutagenic effect of sodium azide was inhibited by tested phenolic acids by about $20-35 \%$. The most effective compound, gallic acid inhibits this effect by $82 \%$ in the concentration of $500 \mu \mathrm{g} / \mathrm{plate}$. The only exception from favourable properties of tested phenolic acids is cichoric acid, which in the contrary significantly increased the mutagenic effect of 5NFAA.

\section{INTRODUCTION}

Phenolic compounds are ubiquitous in plant food, and have been associated with the sensory and nutritional quality of fresh and processed plant foods ${ }^{1}$. In the last years, researchers and food manufacturers are increasingly interested in these compounds, which may be exploited for the development of functional foods or in the chemoprevention. Phenolic compounds have many biological activities; they can act as antioxidants ${ }^{2}$, scavengers of active oxygen species and electrophiles ${ }^{3}$, blockers of nitration ${ }^{4}$ and chelators of metals ${ }^{5}$. They can undergo autooxidation to produce hydrogen peroxide in the presence of metals and are capable of modulating certain cellular enzyme activities $^{6}$. Fortification of foods with materials rich in phenolic compounds has been shown to impart antimutagenic, antiinflammatory and antioxidant properties, which may be exploited for the development of health foods ${ }^{7}$.

Caffeic acid is found naturally in various agricultural products as coffe beans, fruits, vegetables, tobacco leaves, olive oils and wines ${ }^{8}$. This phenolic acid exhibit a cytoprotective effect on endothelial cells against oxidized lowdensity lipoprotein ${ }^{9}$, inhibits the oxidation of lipoprotein exposed to ferrylmyoglobin and recycles $\alpha$-tocopherol from $\alpha$-tocopherol radical ${ }^{10}$. Likewise as other phenolic acids (ferulic and gentisic acids) caffeic acid exhibits protective effect against the genotoxicity of acridin orange and ofloxacin in Salmonella typhimurium ${ }^{11}$ as well as in Euglena gracilis $^{12}$. The therapeutic effect of Echinacea plants has been assigned to the presence of caffeic acid derivatives such as cichoric acid, echinacoside, chlorogenic acid beside of various other components found in the hydroalcoholic extracts ${ }^{13}$. Cichoric acid has been shown to posses phagocytosis stimulatory activity in vitro and in vivo ${ }^{14}$, to inhibit hyaluronidase and to protect colagen type III from free radical-induced degradation ${ }^{14}$. Caffeic and chlorogenic acid possesses inhibitory effect on the mutagenicity of Trp-P-1 and Glu-P-2 (ref. ${ }^{15}$ ). The same phenolic acids may inhibit the formation of mutagenic and carcinogenic $N$-nitroso compounds because they are inhibitors of the $N$-nitrosation reaction in vitro ${ }^{4}$. Tanaka et al. ${ }^{16}$ described the inhibition of 4-nitroquinoline-1-oxideinduced rat tongue carcinogenesis by the caffeic, ellagic, chlorogenic and ferulic acid.

However, not all polyphenols and not all actions of individual polyphenols are necessarily beneficial. Some of them have mutagenic and/or pro-oxidant effect, and they may interfere with essential biochemical pathways ${ }^{17}$. A number of polyphenols, including quercetin, can bind to $\mathrm{DNA}^{18}$ and this direct interaction may be an important mechanism of bacterial mutagenicity. Cichoric acid showed mutagenic activity on Salmonella typhimurium TA98 and TA 100 strains $^{19}$.

In the present study, the plate-incorporation test of the Salmonella mutagenicity assay was used to examine the effect of selected phenolic acids against 5NFAA and sodium azide mutagenicity.

\section{MATERIALS AND METHODS}

Histidin-dependent strain of Salmonella typhimurium TA100 was received from the Collection of Microorganisms, Masaryk University, Brno (Czech Republic). 


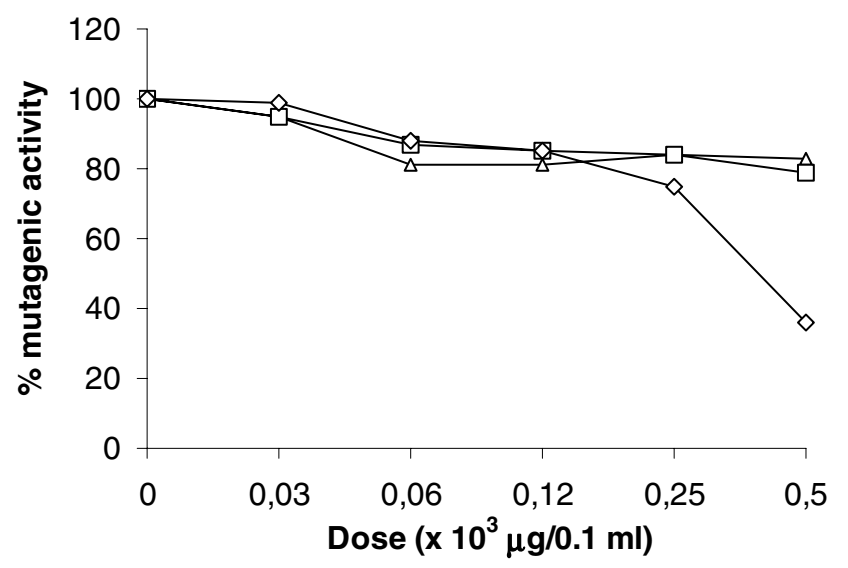

$\neg$ vanillic acid $\neg-$ syringic acid $\diamond$ gallic acid

Fig. 1. Effect of derivatives of hydroxybenzoic acid on 5NFAA-induced mutagenicity in strain TA100 of Salmonella typhimurium. Percent of mutagenic activity $=$ (the number of revertants per plate in the presence of phenolic acid / the number of revertants per plate in the absence of phenolic acid) $\times 100$.

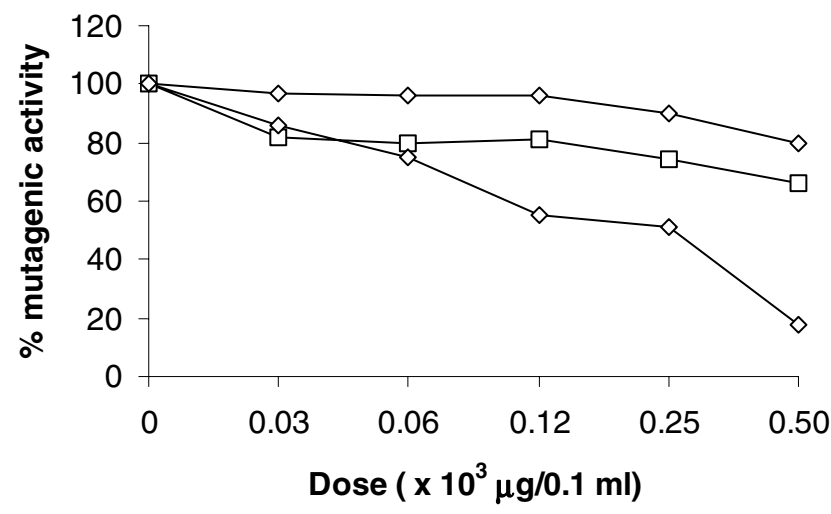

$\prec$ vanillic acid $\neg \square-$ syringic acid $\prec$ gallic acid

Fig. 3. Effect of derivatives of hydroxybenzoic acid on sodium azide-induced mutagenicity in strain TA100 of Salmonella typhimurium. Percent of mutagenic activity expressed as in Fig. 1.

Caffeic acid (3,4-dihydroxycinnamic acid), ferulic acid (4-hydroxy-3-methoxycinnamic acid) and cichoric acid (2,3-O-dicaffeoyltartaric acid) were extracted, isolated and identified according to the methods of Perry et al. ${ }^{20}$ and Wills and Stuart ${ }^{21}$. Vanillic acid (4-hydroxy-3-methoxybenzoic acid), syringic acid (3,5-dimethoxy-4-hydroxybenzoic acid) and gallic acid (3,4,5-trihydroxybenzoic acid) were purchased from Sigma-Aldrich (Germany). The positive mutagens: 3-(5-nitro-2-furyl)acrylic acid (5NFAA) was from Slovakofarma Hlohovec (Slovakia) and sodium azide was purchased from Sigma-Aldrich (Germany).

The inhibitory effect of phenolic acids on mutation induction by several positive mutagens was investigated

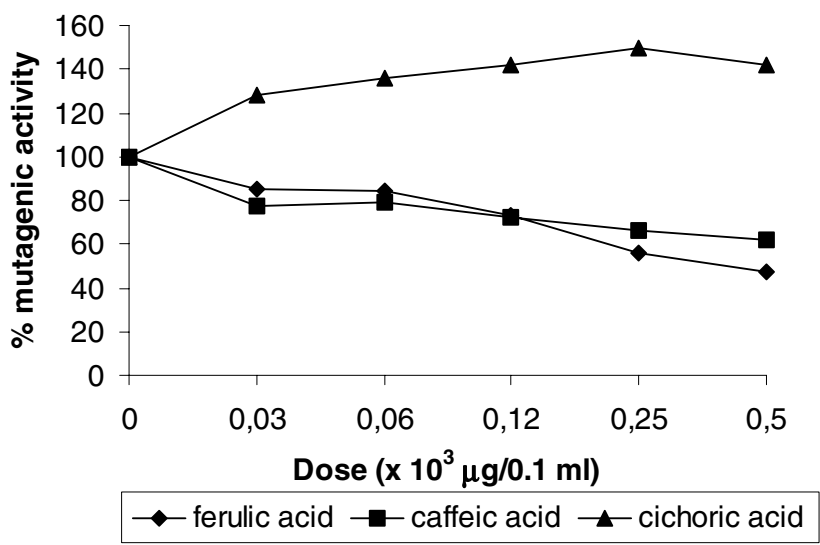

Fig. 2. Effect of derivatives of hydrocinnamic acid on 5NFAA-induced mutagenicity in strain TA100 of Salmonella typhimurium. Percent of mutagenic activity expressed as in Fig. 1.

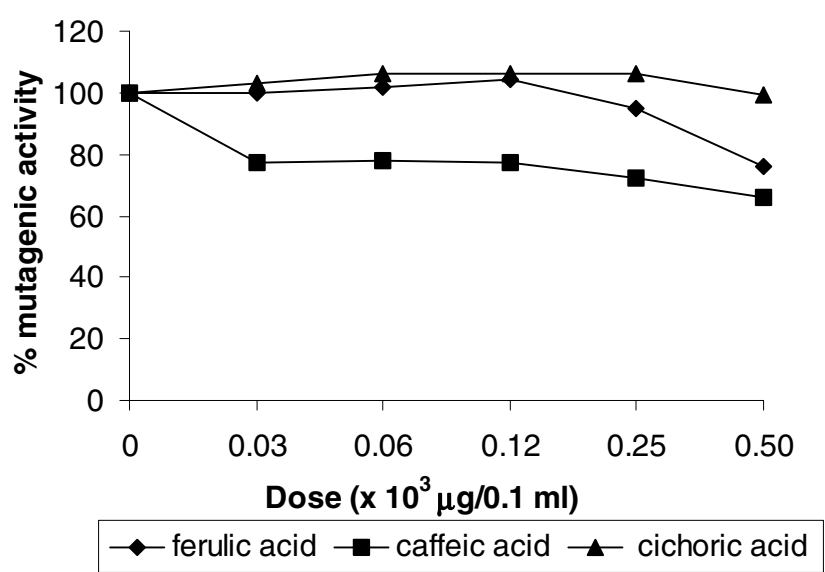

Fig. 4. Effect of derivatives of hydroxycinnamic acid on sodium azide-induced mutagenicity in strain TA100 of Salmonella typhimurium. Percent of mutagenic activity expressed as in Fig. 1.

with Salmonella typhimurium TA100 using pre-incubation method ${ }^{22} .0 .1 \mathrm{ml}$ of the positive mutagen, $0.1 \mathrm{ml}$ of phenolic acid and $0.1 \mathrm{ml}$ of bacterial culture (cultivation for $16 \mathrm{~h}$ at $37^{\circ} \mathrm{C}$, approximate cell density $2-5 \times$ $10^{8}$ cells $/ \mathrm{ml}$ ) were mixed and preincubated at $37^{\circ} \mathrm{C}$ for $30 \mathrm{~min}$. Soft agar $(2 \mathrm{ml})$ was added and the mixture was poured onto minimal agar plates. After $48 \mathrm{~h}$ of incubation at $37^{\circ} \mathrm{C}$ the number of revertants was counted. The results from the antimutagenicity studies represent the mean of three separate experiments, each run in triplicate, and they were statistically evaluated using the Student's $t$-test. Antimutagenicity was expressed as percentage of mutagenicity inhibition following the formula: 


$$
\% \text { mutagenicity }=100-[(\mathrm{X} 1 / \mathrm{X} 2) \times 100]
$$

where $\mathrm{X} 1=$ number of revertants per plate in the presence of mutagen and antimutagen, $\mathrm{X} 2$ = number of revertants per plate in the absence of antimutagen

\section{RESULTS AND DISCUSSION}

Doses of $10 \mu \mathrm{g} /$ plate of 5 NFAA and $5 \mu \mathrm{g} /$ plate of sodium azide were chosen for the antimutagenicity studies since these doses were not toxic and induced $1835 \pm 178$ (5NFAA) and $1263 \pm 153$ (sodium azide) revertants in Salmonella typhimurium TA100. In selecting the concentration of phenolic acids we used the amounts where cell viability was found to be above $90 \%$.

The inhibitory effects of derivatives of hydroxybenzoic acid on the mutagenicity of 5NFAA are illustrated in Fig. 1 . The positive control of mutagen in each case was considered as $100 \%$ mutagenicity. Gallic acid was the only phenolic acid successful in inhibiting the mutagenicity of 5NFAA by more then $50 \%$ at the concentration of $500 \mu \mathrm{g} /$ plate. Vanillic and syringic acids decreased the number of revertants starting from $60 \mu \mathrm{g} /$ plate by about $20 \%$.

It is evident from the Fig. 2 that cichoric acid increased the number of revertants induced by 5NFAA. Ferulic and caffeic acids decreased the mutagenic effect of 5NFAA by about $50 \%$.

Figs. 3 and 4 show the results of inhibitory effect of phenolic acids on the mutagenic effect of sodium azide. With the exception of cichoric acid which has no antimutagenic effect, all tested compounds decreased the number of revertants induced by sodium azide by about 20-35\%. Similarly as in the case of 5NFAA, the effect of sodium azide was significantly reduced by gallic acid and within the concentration tested a marked dose-dependence was found. At the highest used concentration this phenolic acid inhibits mutagenic activity of sodium azide by $82 \%$.

\section{ACKNOWLEDGEMENT}

This work was supported by the Slovak Grant Agency VEGA (Project no. 1/1173/04).

\section{REFERENCES}

1. Ho C. Phenolic compounds in food. In: Ho C, Lee CY, Huang M, editors. Phenolic Compounds in Food and their Effects on Health I. Analysis, Occurrence and Chemistry. Washington, DC: American Chemical Society, 1992. p. 1-7.
2. Kagan VE, Tyurinov YY. (1998) Recycling and redox cycling of phenolic antioxidants. Ann N Y Acad Sci 854, 425-34.

3. Zhou YC, Zheng RL. (1991) Phenolic compounds and an analog as superoxide anion scavengers and antioxidants. Biochem Pharmacol 42,1177-9

4. Kono Y, Shibata H, Kodama Y, Sawa Y. (1995) The suppresion of the N-nitrosating reaction by chlorogenic acid. Biochem J 312, 947-53.

5. Brune M, Rossander L, Hallberg L. (1989) Iron absorption and phenolic compounds: importance of different phenolic structures. Eur J Clin Nutr 43, 547-8.

6. Huang M, Ferraro T. Phenolic compounds in food and cancer prevention. In: Ref. 1, p. 8-35.

7. Friedman MN. (1997) Chemistry, biochemistry and dietary role of potato polyphenols - a review. J Agric Food Chem 45, 1523-40.

8. Woodring PJ, Edwards PA, Chisholm MG. (1990) A HPLC determination of nonflavonoid phenols in Vidal blank wine using electrochemical detection. J Agric Food Chem 38, 729-32.

9. Vieira O, Escarqueil-Blanc I, Meilhac O, Basile JP, Laranjinha J, Almeida L, Salvayre R, Negre-Salvayre A. (1998) Effect of dietary phenolic compounds on apoptosis of human cultured endothelial cells induced by oxidized LDL. Br J Pharmacol 12, 565-73.

10. Laranjinha J, Vieira O, Madeira V, Almeida L. (1995) Two related phenolic antioxidants with opposite effects on vitamin E content in low density lipoprotein oxidized by ferrylmyoglobin: consumption vs. regeneration. Arch Biochem Biophys 323, 373-81.

11. Belicová A, Križková L, Nagy M, Krajčovič J, Ebringer L. (2001) Phenolic acids reduce the genotoxicity of acridine orange and ofloxacin in Salmonella typhimurium TA 102. Folia Microbiol 47, 511-4.

12. Križková L, Nagy M, Polónyi J, Dobias J, Belicová A, Grančai D, Krajčovič J. (2000) Phenolic acids inhibit chloroplast mutagenesis in Euglena gracilis. Mutat Research 469, 107-14.

13. Letchamo W, Livesey J, Arnason TJ, Bergeron C, Krutilina VS. Cichoric acid and isobutylamide content in Echinacea purpurea as influenced by flower developmental stages. In: Janick J, editor. Perspectives on new crops and new uses. Alexandria: ASHS Press, 1999, p. 494-8.

14. Bauer R, Wagner H. Echinacea species as potential immunostimulatory drugs. In: Wagner H, Farnsworth NR, editors. Economic and medicinal plant research. New York: Academic Press, 1991, p. 253-321.

15. Yamada J, Tomita Y. (1996) Antimutagenic activity of caffeic acid and related compounds. Biosci Biotech Biochem 60, 328-9.

16. Tanaka T, Kojima T, Kawamori T, Wang A, Suzui T, Okamoto K, Mori H. (1993) Inhibition of 4-nitroquinoline-1-oxide-induced rat tongue carcinogenesis by the naturally occuring phenolic caffeic, ellagic, chlorogenic and ferulic acids. Carcinogenesis 14, 1321-5.

17. Ferguson LR. (2001) Role of plant polyphenols in genomic stability. Mutat Res 475, 89-111.

18. Alvi NK, Rizvi RY, Hadu SM. (1986) Interaction of quercetin with DNA. Biosci Rep 6, 861-8.

19. Mikulášová M, Vaverková Š, Birošová L, Suchánová M. (2005) Genotoxic effects of the hydroxycinnamic acid derivatives-caffeic, chlorogenic and cichoric acids. Biologia 60 1-5.

20. Perry NB, Burgess EJ, Glennie VI. (2001) Echinacea standardization: analytical methods for phenolic compounds and typical levels in medical species. J Agric Food Chem 49, 1702-6.

21. Wills RBH, Stuart DL. (1999) Alkylamide and cichoric acid levels in Echinacea pupurea grown in Australia. Food Chem 67, 385-8.

22. Maron DM, Ames BN. (1983) Revised method for Salmonella mutagenicity test. Mutat Res 113, 173-215. 